\title{
BMJ Open Efficacy and safety of mesenchymal stem cells for the treatment of patients infected with COVID-19: a systematic review and meta-analysis protocol
}

\author{
Yunhui Chen (D) , ${ }^{1}$ Qing Zhang, ${ }^{1}$ Wei Peng, ${ }^{1}$ Dan Liu, ${ }^{2}$ Yanyan You, ${ }^{2}$ Xinglong Liu, ${ }^{1}$ \\ Songqi Tang, ${ }^{3}$ Tiane Zhang $^{1}$
}

To cite: Chen Y, Zhang Q, Peng W, et al. Efficacy and safety of mesenchymal stem cells for the treatment of patients infected with COVID-19: a systematic review and metaanalysis protocol. BMJ Open 2020;10:e042085. doi:10.1136/ bmjopen-2020-042085

- Prepublication history for this paper is available online. To view these files, please visit the journal online (http://dx.doi. org/10.1136/bmjopen-2020042085).

YC and QZ contributed equally.

Received 24 June 2020 Revised 09 September 2020 Accepted 05 October 2020

Check for updates

(c) Author(s) (or their employer(s)) 2020. Re-use permitted under CC BY-NC. No commercial re-use. See rights and permissions. Published by BMJ.

${ }^{1}$ Chengdu University of Traditional Chinese Medicine, Chengdu, China

${ }^{2}$ West China Hospital, Sichuan University, Chengdu, China

${ }^{3}$ Hainan Medical University, Haikou, China

\section{Correspondence to}

Professor Songqi Tang; tangsongqi@foxmail.com and Professor Tiane Zhang; zhte2003@cdutcm.edu.cn

\section{ABSTRACT}

Introduction To date, no specific antivirus drugs or vaccines have been available to prevent or treat the COVID-19 pandemic. Mesenchymal stem cell (MSC) therapy may be a promising therapeutic approach that reduces the high mortality in critical cases. This protocol is proposed for a systematic review and meta-analysis that aims to evaluate the efficacy and safety of MSC therapy on patients with COVID-19.

Methods and analysis Ten databases including PubMed, EMBASE, Cochrane Library, CINAHL, Web of Science, Chinese National Knowledge Infrastructure (CNKI), Chinese Scientific Journals Database (VIP), Wanfang database, China Biomedical Literature Database (CBM) and Chinese Biomedical Literature Service System (SinoMed) will be searched from inception to 1 December 2020. All published randomised controlled trials, clinical controlled trials and case series that meet the prespecified eligibility criteria will be included. The primary outcomes include mortality, incidence and severity of adverse events, respiratory improvement, days from ventilator, duration of fever, progression rate from mild or moderate to severe, improvement of such serious symptoms as difficulty breathing or shortness of breath, chest pain or pressure, and loss of speech or movement, biomarkers of laboratory examination and changes in CT. The secondary outcomes include dexamethasone doses and quality of life. Two reviewers will independently perform study selection, data extraction and assessment of bias risk. Data synthesis will be conducted using RevMan software (V.5.3.5). If necessary, subgroup and sensitivity analysis will be performed. Grading of Recommendations Assessment, Development and Evaluation system will be used to assess the strength of evidence.

Ethics and dissemination Ethical approval is not necessary since no individual patient or privacy data have been collected. The results of this review will be disseminated in a peer-reviewed journal or an academic conference presentation.

PROSPERO registration number CRD42020190079.

\section{INTRODUCTION}

\section{Description of the condition}

COVID-19, an infectious disease caused by SARS-CoV-2 (formerly termed as 2019-nCoV),
Strengths and limitations of this study

- This systematic review protocol follows the Preferred Reporting Items for Systematic Review and MetaAnalysis Protocols guidelines.

- This systematic review, to the best of our knowledge, will be the first to explore the efficacy and safety of mesenchymal stem cell (MSC) therapy for the treatment of patients infected with COVID-19.

- The study will systematically review quantitative data from various medical databases for an in-depth interpretation of the efficacy and safety of MSC therapy on patients with COVID-19.

- The potential for low and inconsistent quality in the reporting of process evaluations, the publication bias and the methodological quality of the grey literature found may be the limitations of the study. Other potential limitation might be whether a sufficient number of trials would be completed such that patient data are widely available to make interpretations or draw conclusions.

has been sweeping the world. ${ }^{1}$ However, to date, no efficient vaccines and specific antiviral medications are available to halt the pandemic. As of 24 June 2020, a total of 9110 186 confirmed cases of COVID-19 have been documented globally in 216 countries, areas or territories with 473061 deaths. $^{2}$ Notably, approximately $15 \%$ of the infected patients develop severe pneumonia and 5\% progress to acute respiratory distress syndrome (ARDS), septic shock and multiorgan failure eventually. ${ }^{34}$ High mortality rate was observed in critically ill patients and has prompted an urgent need for treatments that can address the critical cases and prevent fatal outcomes. ${ }^{56}$

Description of the intervention

Currently, mesenchymal stem cell (MSC)based treatment has been proposed as a promising therapeutic approach for patients with COVID-19. ${ }^{7}$ MSCs are multipotent cells 
that can be obtained from various tissues including preferably bone marrow, adipose tissue, placenta, umbilical cord and dental pulp. The safety and effectiveness of MSC therapy have been well documented in several clinical studies including ARDS, bronchopulmonary dysplasia and cardiovascular diseases. ${ }^{8-12}$ In a recent case study, a 65-year-old critically ill ventilator-ridden patient with COVID-19 was treated with allogeneic human umbilical cord MSCs (three infusions of $5 \times 10^{7}$ cells at an interval of 3 days) and the patient was off the ventilator and able to walk after the second infusion. No obvious side effects were observed. ${ }^{13}$ In another study, seven patients with COVID-19 (one critically severe, four severe and two mild to moderate) received a single intravenous administration of MSCs at a dose of $1 \times 10^{6}$ cells $/ \mathrm{kg}$ body weight, all patients had significant improvements in clinical symptoms 2 days after the transplantation, one severe and two mild patients were discharged from the hospital by day 10 and a 14-day follow-up showed no adverse effects. ${ }^{14}$ These completed clinical trials provided limited but promising evidence that the use of MSC therapy might be efficient in the treatment of COVID-19. The US Food and Drug Administration authorised compassionate use of MSC therapy in patients with an extremely dismal prognosis. Recently, 45 clinical trials of MSCs as a new treatment for COVID-19 have been registered on ChiCTR (http://www. chictr.org.cn) and ClinicalTrials.gov (https://clinicaltrials.gov). Several trials are underway that may provide more evidence to evaluate the safety and effectiveness of MSC therapy for COVID-19.

\section{How the intervention might work}

A growing body of research has interpreted the potential mechanism underlying the therapeutic effect of MSCs on COVID-19. SARS-CoV-2 cell entry depends on the ACE2 and the transmembrane serine protease 2 (TMPRSS2), gene expression profiling exhibited that transplanted MSCs were ACE2 and TMPRSS2 and had a natural immunity to the COVID-19 infection. ${ }^{14}{ }^{15}$ Virus-induced cytokine storm is considered as the hallmark of SARS-CoV-2 pathogenesis and has been reported to induce ARDS and multiorgan failure, leading to death in patients with COVID-19. ${ }^{16}$ To prevent or attenuate the cytokine storm is pivotal to halt the pandemic. Compelling studies have demonstrated that MSCs have potent and broad immunomodulatory and anti-inflammatory effects. Such processes include the regulation of T-cell function, proliferation and differentiation of $\mathrm{B}$ cells, influence of innate immune cells (such as macrophages and dendritic cells), decrease of proinflammatory cytokines (such as interleukin 1 (IL-1), IL-6, interferon (IFN) and tumour necrosis factoralpha), increase of anti-inflammatory cytokines (such as IL-4, IL-5 and IL-10) and peripheral lymphocytes, and decline of overactivated cytokine-secreting immune cells (such as CXCR3+ CD4+ T cells, CXCR3+ CD8+ T cells and CXCR3+ natural killer cells). ${ }^{14}$ 17-23 Besides, MSCs inhibit bacterial growth, enhance the restoration of injured alveolar epithelial cells, improve pulmonary microenvironment, alleviate pulmonary fibrosis and enhance pulmonary function. ${ }^{24}{ }^{25}$ Kyoto Encyclopedia of Genes and Genomes analysis implied that MSCs were involved in antiviral pathways. ${ }^{14}$ Hence, MSC therapy may improve the outcome of patients with COVID-19 through immunomodulation, regulating the inflammatory response and promoting tissue repair.

\section{Why it is important to perform this review}

The pandemic COVID-19 caused by SARS-CoV-2 has been sweeping the world. MSC therapy has been proposed as a safe, effective and promising approach to treat COVID19 , especially for severe or critical cases. It is important to perform a systematic review and meta-analysis to evaluate its efficacy and safety. In this systematic review, all potential randomised controlled trials (RCTs), clinical controlled trials (CCTs) and case series regarding MSCs for the treatment of COVID-19 will be fully considered and synthesised without language or publication restrictions. The findings of this study may yield helpful evidence for the patients, clinicians, investigators and policymakers concerned about the efficacy and safety of MSC therapy on COVID-19.

\section{Objectives}

This systematic review and meta-analysis aims to assess the evidence for the effectiveness and safety of MSC therapy for COVID-19.

\section{METHODS AND ANALYSIS}

A statement: this is to clarify that due to the similarity of the methods, there is some overlap with our previous publication. $^{26}$

\section{Criteria for including studies for this review \\ Types of studies}

This review will include RCTs, CCTs and case series of MSCs treatment for COVID-19. Snowballed papers from references will also be included. Animal-based research and literature review will be excluded.

\section{Types of participants}

This review will include patients diagnosed with COVID-19 using any recognised diagnostic criteria regardless of the age, gender, and source of cases and the duration and severity of the disease. Patients infected with adenovirus, rhinovirus, human metapneumovirus, and so on will be excluded.

\section{Types of interventions}

The intervention group will receive treatment of MSCs and standard care. This review will impose no restriction on standard care regimens (including supportive treatment, IFN- $\alpha$, lopinavir or ritonavir).

\section{Types of comparator(s)/control}

The control group will receive the same treatment of standard care regimen as the intervention group in the 
same original study. This review will impose no restrictions regarding standard care regimen.

Types of outcome measures

\section{Primary outcomes}

The primary outcomes include mortality, incidence and severity of adverse events, respiratory improvement, days from ventilator, duration of fever, progression rate from mild or moderate to severe, improvement of such serious symptoms as difficulty breathing or shortness of breath, chest pain or pressure, and loss of speech or movement, biomarkers of laboratory examination and changes in CT.

\section{Secondary outcomes}

Secondary outcomes include dexamethasone doses and quality of life.

\section{Search methods for identification of studies \\ Electronic searches}

The following 10 databases will be searched from the inception to 1 December 2020: PubMed, EMBASE, Cochrane Library, CINAHL, Web of Science, Chinese National Knowledge Infrastructure (CNKI), Chinese Scientific Journals Database (VIP), Wanfang database, China Biomedical Literature Database (CBM) and Chinese Biomedical Literature Service System (SinoMed). The literature will be searched by two reviewers independently. Any discrepancies will be resolved by consultation with a third reviewer. Manual search will be performed on the reference lists of included studies for relevant publications.

The reviewers will use a combination of the following terms to conduct the electronic search: novel coronavirus, severe acute respiratory syndrome coronavirus 2, SARS-CoV-2, COVID-19, COVID19, 2019-nCoV, mesenchymal stem cells, mesenchymal stromal cells, MSCs, stem cells, SCs, randomised controlled trial, controlled clinical trial, randomised, randomly, trials, case series, CCT and RCT. Table 1 presents the search strategy for PubMed, and the reviewers will modify the strategy correspondingly on the requirement of other databases.

\section{Searching other resources}

The reviewers will also search the studies from ClinicalTrials.gov (http://clinicaltrials.gov), Chinese Clinical Trial Registry (http://www.chictr.org/cn/) and WHO International Clinical Trial Registry Platform (https:// www.who.int/ictrp/en/). Grey literature such as guidelines, research and committee reports, government reports and conference papers will be obtained from WHO, US National Library of Medicine, Chinese Center for Disease Control and Prevention and online official news websites. The reviewers will manually review the reference lists of the retrieved articles for further additional trials. For incomplete data, the corresponding author will be contacted.

\begin{tabular}{|c|c|}
\hline No & Search terms \\
\hline \#1 & Novel coronavirus \\
\hline \#2 & Severe acute respiratory syndrome coronavirus 2 \\
\hline$\# 3$ & SARS-CoV-2 \\
\hline \#4 & COVID-19 \\
\hline \#5 & COVID19 \\
\hline \#6 & 2019-nCoV \\
\hline \#7 & OR/\#1-\#6 \\
\hline \#8 & Stem cells \\
\hline$\# 9$ & SC \\
\hline$\# 10$ & Mesenchymal stem cells \\
\hline$\# 11$ & Mesenchymal stromal cells \\
\hline$\# 12$ & MSCs \\
\hline$\# 13$ & $\mathrm{OR} / \# 8-\# 12$ \\
\hline$\# 14$ & \#7 AND \#13 \\
\hline$\# 15$ & Randomised controlled trial \\
\hline$\# 16$ & Controlled clinical trial \\
\hline$\# 17$ & Randomised* \\
\hline$\# 18$ & Randomly* \\
\hline \#19 & Trials \\
\hline \#20 & Case series \\
\hline \#21 & СCT \\
\hline \#22 & $\mathrm{RCT}$ \\
\hline \#23 & OR/\#14-\#22 \\
\hline \#24 & \#7 AND \#14 AND \#23 \\
\hline
\end{tabular}

${ }^{*}$ Represent one or more characters of all characters.

CCT, clinical controlled trial; MSC, mesenchymal stem cell; RCT, randomised controlled trial; SC, stem cell.

\section{Data collection and analysis}

\section{Selection of studies}

Two reviewers will independently conduct screening, study selection and data extraction. The reviewers will import the literature obtained into EndNote V.X9, screen the title and abstract and exclude the duplications and studies that did not meet the inclusion criteria. The final included studies will be determined after reading the full text of the remaining studies. If the full text is not available, the corresponding author of the original study will be contacted. A third reviewer will be consulted for arbitrating any disagreements. A Preferred Reporting Items for Systematic Reviews and Meta-Analyses flow chart is presented to summarise the entire process of the study selection (figure 1).

\section{Data extraction and management}

Two reviewers will independently extract the following data from eligible studies using a prespecified data acquisition form: reference ID, author information, year of publication, study type, study design, setting of study, sample size, participant characteristics (age, gender, 


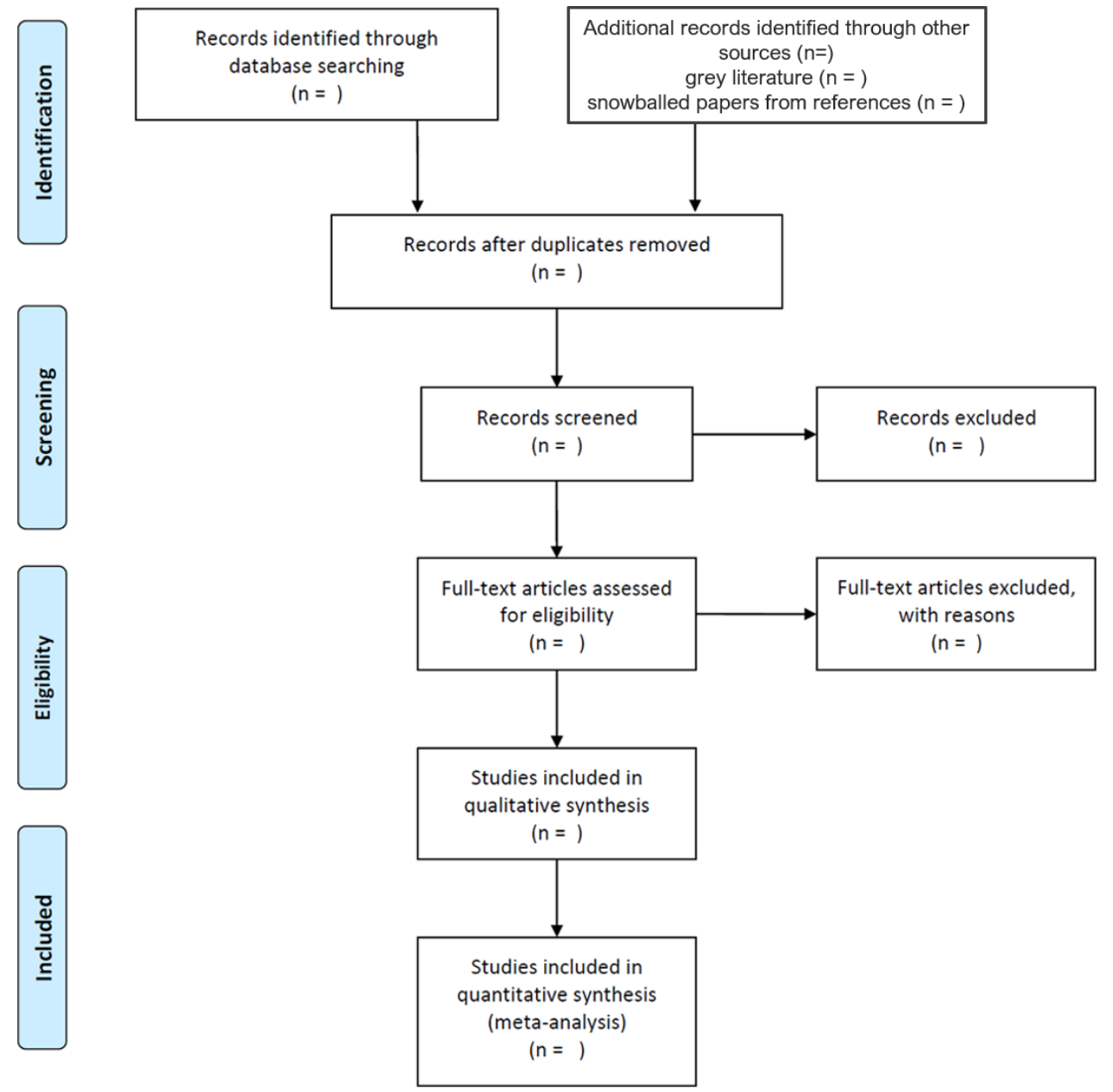

Figure 1 Flow chart of study selection.

duration and severity of illness, laboratory test, CT scan, and so on), MSC intervention group and control group (details of randomisation, blinding, allocation, intervention approach and duration) and primary and secondary outcomes at all reported time points. A third reviewer will be consulted to solve any inconsistency between the two reviewers. A crosscheck will be performed on all data before transferring to RevMan software (V.5.3).

\section{Assessment of risk of bias}

The Cochrane Risk of Bias Assessment Tool will be used by two reviewers to independently evaluate the risk of publication bias for all the included studies in eight domains, namely randomisation sequence generation, randomisation allocation concealment, blinding of participants, blinding of personnel, blinding of outcome assessors, incomplete outcome data, selective reporting bias and other bias. Each domain will be graded as high, unclear or low risk of bias. For unclear domain, the reviewers will contact the corresponding authors of the original study. Any inconsistency will be solved by discussion with a third reviewer.

\section{Measures of treatment effect}

Two reviewers will independently synthesise and statistically analyse efficacy data using RevMan V.5.3. The reviewers will use a risk ratio or OR with $95 \%$ CIs for dichotomous data and a mean difference or standard mean difference (SMD) with 95\% CIs for continuous data. When different assessment tools are employed, SMD will be used.

\section{Dealing with missing data}

If the required data are unclear or unavailable, the original study's corresponding author will be contacted by email or telephone. If data are still unattainable, the reviewers will exclude the study concerned from the analysis. To address the potential effects of missing data, a sensitivity analysis will be conducted.

\section{Assessment of heterogeneity}

$\chi^{2}$ test and $\mathrm{I}^{2}$ statistic will be employed to investigate statistical heterogeneity. The fixed effects model will be used for a low heterogeneity $\left(\mathrm{I}^{2}<50 \%\right)$ and the random effects model will be applied when the heterogeneity is moderate $\left(50 \%<\mathrm{I}^{2}<75 \%\right)$. Meta-analysis will not be conducted if the heterogeneity is remarkably high $\left(\mathrm{I}^{2}>75 \%\right)$.

\section{Assessment of reporting biases}

This review will perform funnel plots to assess potential reporting bias when more than 10 eligible studies are included. Additionally, the Egger regression and Begg 
correlation test will be used to identify the funnel plot asymmetry.

\section{Data synthesis}

Following the Cochrane guideline, the reviewers will use the fixed effects model for the pooled data when heterogeneity is deemed low and the random effects model if heterogeneity is moderate. ${ }^{27}$ This review will perform subgroup analysis or meta-regression to evaluate the potential sources and provide reasonable explanations when heterogeneity is considerably high. $\mathrm{P}<0.05$ is deemed statistically significant. If the meta-analysis is unfeasible, the results will be described narratively.

\section{Subgroup analysis and investigation of heterogeneity}

If feasible, subgroup analyses will be performed in terms of the disease severity of included patients, duration of disease, routes of administration, dosage and origin of MSCs. Subgroup analyses will be conducted to interpret the heterogeneity.

\section{Sensitivity analysis}

If feasible, this review will conduct sensitivity analysis to assess the robustness of the pooled effects of the included studies given the impact of such variables as sample size, methodological quality, missing data or high risk of bias.

\section{Summary of evidence}

This review will use the Cochrane Collaboration Network Grading of Recommendations Assessment, Development and Evaluation to grade the quality of evidence as very low, low, moderate or high. ${ }^{28}{ }^{29}$ The quality of evidence of a specific study will be evaluated based on the risk of bias, imprecision, inconsistency, indirectness, publication bias, effect size or dose-response relation. The findings will be presented in A Summary of Finding table. Any discrepancy will be resolved by discussion or arbitrated by a third reviewer.

\section{Patient and public involvement \\ No patient was involved.}

\section{Ethics and dissemination}

Ethical approval is not necessary as no individual patient or privacy data will be collected. The results of this study will be disseminated in a peer-reviewed journal or an academic conference presentation.

\section{DISCUSSION}

This meta-analysis will analyse the efficacy and safety of MSC therapy for treatment of patients with COVID-19 using a structured and valid methodology. Conclusions drawn from this study may provide useful information and facilitate the decision-making process of patients, clinicians, investigators and policymakers. The process of performing this review will include identification, study inclusion, data extraction and data synthesis. If this protocol needs to be amended, we will provide the date of each amendment with a statement of the changes and corresponding reasons. For the ongoing incoming literature, this meta-analysis will be regularly updated with new incoming data from randomised studies.

Contributors YC, QZ, TZ and ST conceived and designed the study. The protocol was drafted by YC, QZ, XL and ST and revised by YC, WP and TZ. YC, WP, ST and TZ designed the search strategies. $\mathrm{XL}$ and $\mathrm{DL}$ performed searching, data curation and assessment independently. QZ and YY analysed and interpreted the data. ST, TZ, $\mathrm{XL}$ and $\mathrm{YC}$ arbitrated the disagreement if there was anything during the review. All authors have read and approved the publication of the protocol.

Funding This study was funded by the International Cooperation and Exchange Project of Sichuan Provincial Science and Technology Department (grant number 2017HH0004), the National Natural Science Foundation of China (grant numbers 81603537,81860840 and 81860816) and the Youth Scholar Project of Chengdu University of Traditional Chinese Medicine (grant number QNXZ2019043).

Competing interests None declared.

Patient and public involvement Patients and/or the public were not involved in the design, or conduct, or reporting, or dissemination plans of this research.

Patient consent for publication Not required.

Open access This is an open access article distributed in accordance with the Creative Commons Attribution Non Commercial (CC BY-NC 4.0) license, which permits others to distribute, remix, adapt, build upon this work non-commercially, and license their derivative works on different terms, provided the original work is properly cited, appropriate credit is given, any changes made indicated, and the use is non-commercial. See: http://creativecommons.org/licenses/by-nc/4.0/.

ORCID iD

Yunhui Chen http://orcid.org/0000-0002-3555-8018

\section{REFERENCES}

1 Lu R, Zhao X, Li J, et al. Genomic characterisation and epidemiology of 2019 novel coronavirus: implications for virus origins and receptor binding. Lancet 2020;395:565-74.

2 World Health Organization. Rolling updates on coronavirus disease (COVID-19), 2020. Available: https://www.who.int/emergencies/ diseases/novel-coronavirus-2019/events-as-they-happen

3 Huang C, Wang Y, Li X, et al. Clinical features of patients infected with 2019 novel coronavirus in Wuhan, China. Lancet 2020;395:497-506.

$4 \mathrm{Xu} \mathrm{Z}$, Shi L, Wang Y, et al. Pathological findings of COVID-19 associated with acute respiratory distress syndrome. Lancet Respir Med 2020;8:420-2.

5 Bhatraju PK, Ghassemieh BJ, Nichols M, et al. Covid-19 in critically ill patients in the seattle region - case series. $N$ Engl J Med 2020;382:2012-22.

6 Zumla A, Hui DS, Azhar El, et al. Reducing mortality from 2019-nCoV: host-directed therapies should be an option. Lancet 2020;395:e35-6.

7 Golchin A, Farahany TZ. Biological products: cellular therapy and FDA Approved products. Stem Cell Rev Rep 2019;15:166-75.

8 Golchin A, Farahany TZ, Khojasteh A, et al. The clinical trials of mesenchymal stem cell therapy in skin diseases: an update and Concise review. Curr Stem Cell Res Ther 2019;14:22-33.

9 Matthay MA, Calfee CS, Zhuo H, et al. Treatment with allogeneic mesenchymal stromal cells for moderate to severe acute respiratory distress syndrome (start study): a randomised phase $2 \mathrm{~A}$ safety trial. Lancet Respir Med 2019;7:154-62.

10 Chen J, Hu C, Chen L, et al. Clinical study of mesenchymal stem cell treatment for acute respiratory distress syndrome induced by epidemic influenza A (H7N9) infection: a hint for COVID-19 treatment. Engineering 2020;6:1153-61.

11 Namba F. Mesenchymal stem cells for the prevention of bronchopulmonary dysplasia. Pediatr Int 2019;61:945-50.

12 Suvakov S, Richards C, Nikolic V, et al. Emerging therapeutic potential of mesenchymal stem/stromal cells in preeclampsia. Curr Hypertens Rep 2020;22:37.

13 Liang B, Chen J, Li T, et al. Clinical remission of a critically ill COVID-19 patient treated by human umbilical cord mesenchymal stem cells: a case report. Medicine 2020;99:e21429.

14 Leng Z, Zhu R, Hou W, et al. Transplantation of ACE2- Mesenchymal Stem Cells Improves the Outcome of Patients with COVID`19 Pneumonia. Aging Dis 2020;11:216-28. 
15 Hoffmann M, Kleine-Weber H, Schroeder S, et al. SARS-CoV-2 cell entry depends on ACE2 and TMPRSS2 and is blocked by a clinically proven protease inhibitor. Cell 2020;181:271-80.

16 Soy M, Keser G, Atagündüz P, et al. Cytokine storm in COVID-19: pathogenesis and overview of anti-inflammatory agents used in treatment. Clin Rheumatol 2020;39:2085-94.

17 Chen Q-H, Wu F, Liu L, et al. Mesenchymal stem cells regulate the Th17/Treg cell balance partly through hepatocyte growth factor in vitro. Stem Cell Res Ther 2020;11:91.

18 Luz-Crawford P, Hernandez J, Djouad F, et al. Mesenchymal stem cell repression of Th17 cells is triggered by mitochondrial transfer. Stem Cell Res Ther 2019;10:232.

19 Cho K-A, Lee J-K, Kim Y-H, et al. Mesenchymal stem cells ameliorate B-cell-mediated immune responses and increase IL-10expressing regulatory $\mathrm{B}$ cells in an EBI3-dependent manner. Cell Mol Immunol 2017;14:895-908.

20 de Witte SFH, Luk F, Sierra Parraga JM, et al. Immunomodulation by therapeutic mesenchymal stromal cells (MSC) is triggered through phagocytosis of MSC by monocytic cells. Stem Cells 2018;36:602-15.

21 Reis M, Mavin E, Nicholson L, et al. Mesenchymal stromal cellderived extracellular vesicles attenuate dendritic cell maturation and function. Front Immunol 2018;9:2538.
22 Shi Y, Wang Y, Li Q, et al. Immunoregulatory mechanisms of mesenchymal stem and stromal cells in inflammatory diseases. Nat Rev Nephrol 2018;14:493-507.

23 Uccelli A, de Rosbo NK. The immunomodulatory function of mesenchymal stem cells: mode of action and pathways. Ann NY Acad Sci 2015;1351:114-26.

24 Krasnodembskaya A, Song Y, Fang X, et al. Antibacterial effect of human mesenchymal stem cells is mediated in part from secretion of the antimicrobial peptide LL-37. Stem Cells 2010;28:2229-38.

25 Rajarshi K, Chatterjee A, Ray S. Combating COVID-19 with mesenchymal stem cell therapy. Biotechnol Rep 2020;26:e00467.

26 Liu D, You Y, Chen Y, et al. Efficacy of integrative traditional Chinese and Western medicine for the treatment of patients infected with 2019 novel coronavirus (COVID-19): a protocol for systematic review and meta analysis. Medicine 2020;99:e20781.

27 Chandler J, Higgins JPT, Deeks JJ. Cochrane handbook for systematic reviews of interventions version 5.2.0: cochrane community. 2nd Edition. Chichester (UK: John Wiley \& Sons, 2019.

28 Guyatt GH, Oxman AD, Vist GE, et al. Grade: an emerging consensus on rating quality of evidence and strength of recommendations. $B M J$ 2008;336:924-6.

29 Guyatt GH, Oxman AD, Schünemann HJ, et al. Grade guidelines: a new series of articles in the Journal of clinical epidemiology. $J$ Clin Epidemiol 2011;64:380-2. 